\title{
FEASIBILITY STUDY OF RANS IN PREDICTING PROPELLER CAVITATION IN BEHIND-HULL CONDITIONS
}

\author{
Yuxin Zhang \\ Shanghai Merchant Ship Design and Research Institute, China \\ Xiao-ping Wu \\ Shanghai Merchant Ship Design and Research Institute, China \\ Ming-yan Lai \\ Shanghai Merchant Ship Design and Research Institute, China \\ Guo-ping Zhou \\ Shanghai Merchant Ship Design and Research Institute, China \\ Jie Zhang \\ Shanghai Maritime University, China
}

\begin{abstract}
The propeller cavitation not only affects the propulsive efficiency of a ship but also can cause vibration and noise. Accurate predictions of propeller cavitation are crucial at the design stage. This paper investigates the feasibility of the Reynolds-averaged Navier-Stokes (RANS) method in predicting propeller cavitation in behind-hull conditions, focusing on four aspects: (i) grid sensitivity; (ii) the time step effect; (iii) the turbulence model effect; and (iv) ability to rank two slightly different propellers. The Schnerr-Sauer model is adopted as the cavitation model. A model test is conducted to validate the numerical results. Good agreement on the cavitation pattern is obtained between the model test and computational fluid dynamics. Two propellers are computed, which have similar geometry but slightly different pitch ratios. The results show that RANS is capable of correctly differentiating the cavitation patterns between the two propellers in terms of the occurrence of face cavitation and the extent of sheet cavitation; moreover, time step size is found to slightly affect sheet cavitation and has a significant impact on the survival of the tip vortex cavitation. It is also observed that grid refinement is crucial for capturing tip vortex cavitation and the two-equation turbulence models used - realizable $k-\varepsilon$ and shear stress transport (SST) $k-\omega$-yield similar cavitation results.
\end{abstract}

Keywords: RANS,Computational Fluid Dynamics (CFD),propeller cavitation,turbulence model,grid sensitivity

\section{INTRODUCTION}

The cavitation caused by a marine propeller operating in a ship's wake is the source of many negative effects, including thrust loss, erosion, structural vibration and noise. Therefore, it is crucial for ship designers to estimate the negative effect of cavitation at the design stage, especially for luxury cruise ships, rescue ships and high-speed container vessels. Generally, there are two ways to predict a propeller's cavitation: model tests and numerical simulations. The former scales down the ship and propeller to model size so that cavitation can be tested in cavitation tunnels $[1,2,3]$. However, it is expensive and requires considerable time to execute, limiting its application at the propeller design stage. Instead, it tends to be adopted for final verification.

Numerical simulations are more feasible in terms of efficiency and cost, among which the boundary element method (BEM), computational fluid dynamics (CFD) and their combinations are most commonly used $[4,5]$. BEM is widely used in predicting propeller performance because it is quite efficient, while CFD is a promising tool as it provides a high-fidelity simulation, yielding cavitating flow details. In recent years, CFD has been increasingly applied to predict and analyze propeller cavitating flows in both open-water and behind-hull conditions. For instance, Yilmaz et al. (2018) [6] carried out a CFD study on propeller 
cavitation in the presence of a rudder. Wang et al. (2018) [7] numerically analyzed the cavitating flow of an oscillating propeller. Sakamoto et al. (2018) [8] investigated near-field cavitation noise by combining CFD with a semi-empirical method. Asnaghi et al. (2018) [3] analyzed the cavitation inception of high-skewed low-noise propellers. Gaggero et al. (2017) [9] discussed the potential of CFD in propeller cavitation optimization at the design phase. The above works were carried out in open-water conditions. In behind-hull conditions, the cavitating flow is more complicated due to the presence of the hull wake. Nevertheless, some researchers have produced work in this direction. Hur et al. (2018) [10] simulated sheet cavitation on the blade surface, noting good agreement between their CFD simulation and model test in terms of cavitation patterns and pressure fluctuations. Ando et al. (2018) [11] presented a hybrid method comprising CFD analysis and a bubble dynamics model to estimate cavitation noise, finding good agreement with their experimental results. Zheng et al. (2019) [12] simulated the cavitation of a 30,000 DWT bulk carrier using Reynolds-averaged Navier-Stokes (RANS) combined with shear stress transport (SST) $k$ - $\omega$, revealing good agreement in the cavitation pattern between their experiment results and CFD. Moreover, some studies utilized advanced turbulence models. Usta and Korkut (2018) [13] applied SST $k$ - $\omega$-based detached eddy simulation (DES) to predict the cavitation of a propeller in open water, observing good agreement between CFD and their experiment results. Cheng et al. (2020) ${ }^{[14]}$ studied the tip-leakage cavitating flow of a hydrofoil based on large eddy simulation (LES), focusing on the effects of cavitation on vorticity and turbulence, finding that LES was able to capture complicated flow details in cavitating flows.

Nevertheless, CFD is rarely applied at the propeller design stage, mainly due to its high computational cost. To optimize cavitation performance, numerous cases are simulated to identify the optimal propeller geometry. Therefore, it is important to find a balance between computational accuracy and time. In this regard, advanced turbulence models such as DES and LES are not feasible even though they can offer better predictions of unsteady flow features, while RANS is a promising tool considering its efficiency and accuracy.

Previous RANS studies in cavitation prediction have tended to focus on comparing experimental and simulation results. By contrast, few works have considered the ability of RANS to correctly rank propellers with slightly different geometries, which is crucial for CFD to be applied in propeller optimization. Gaggero et al. (2014) [15] investigated two ducted propellers in open water, finding that RANS provided good predictions of tip leakage vortex cavitation and correctly differentiated the two ducted propellers. Nevertheless, the cavitation of a marine propeller may exhibit a variety of patterns depending on the cavitation's location or physical appearance, such as sheet cavitation, tip and hub vortex cavitation and cloud cavitation. The ability of CFD to capture these cavitation patterns and correctly rank propellers with different cavitation patterns is still largely unknown.
The aim of this paper is to analyze the feasibility of RANS in cavitation predication and to provide a reference for propeller designers on how to appropriately choose a mesh parameter, time step size and turbulence model to capture different types of cavitation. We adopt two propellers with similar geometries but slightly contrasting pitch ratio distributions and different cavitation behaviours in terms of sheet, tip vortex and face cavitation. By simulating the two propellers, the ability of RANS to capture different types of cavitation and correctly rank similar propellers can be revealed. To validate the CFD results, a model test is carried out in a cavitation tunnel. It is known that grid number and time step are the factors that significantly affect computational time. However, the optimal grid density and time step size are not known for capturing different types of cavitation. Therefore, the effects of grid and time step are discussed in this work. Moreover, the turbulence effect is discussed against the two most commonly used twoequation turbulence models: realizable $k-\varepsilon$ and SST $k-\omega$.

\section{PROPELLER GEOMETRY AND EXPERIMENT SET-UP}

Two propellers designed for a container vessel 1900 TEU, with similar geometries but slightly different pitch ratios, are investigated here. Table 1 summarizes the principle dimensions of the two propellers. The blade section type is NACA 66. A cavitation model test is carried out in the cavitation tunnel of the China Ship Scientific Research Center (CSSRC) to provide validation data for CFD simulations. Fig. 1 shows the propeller models used in the cavitation test. The scale ratio is $1 / 26.8283$. The dimensions of the tunnel at the measuring segment are $10.5 \mathrm{~m}(\mathrm{~L}) \times 2.2 \mathrm{~m}(\mathrm{~W}) \times 2.0 \mathrm{~m}(\mathrm{H})$. The set-up of the model test is shown in Fig. 2. Two cameras are installed in the stern of the hull to take photos during the experiment. The free surface is not considered in the model test. The stern wave effect is taken into account by increasing the model draft by $0.05 \mathrm{~m}$.

Traditionally, a high propeller revolution speed is used in cavitation tests to eliminate the Reynolds number effect as far as possible. The propeller revolution speed is set at 28 revolutions per second (rps). The inflow velocity is adjusted to ensure the propeller is operating at the target thrust load coefficient, which is defined as:

$$
K_{T}=\frac{T}{\rho n^{2} D^{4}}
$$

where $T$ is the thrust, $\rho$ is the water density, $n$ is the propeller rotation speed and $D$ is the propeller diameter. $T$ corresponds to the propeller thrust at the target ship speed; the function between $\mathrm{T}$ and ship speed is determined by the self-propulsion test. For the ship investigated, the ship speed at design draft with given power is $18.92 \mathrm{kts}$ and the corresponding $K_{T}$ is 0.167 , according to the result of the self-propulsion test. This operating condition is investigated in the cavitation 
test. Cavitation number, noted as, is an important factor for cavitating flow. It is defined here as:

$$
\sigma_{n}=\frac{P-P_{v}}{\frac{1}{2} \rho(\pi n D)^{2}}
$$

where $P$ is the static pressure and $P_{v}$ is the saturation pressure. The cavitation number is the difference between $P$ and $P$, divided by the dynamic pressure of the incoming flow. The cavitation number corresponding to the above-mentioned operating condition is $\sigma_{\mathrm{n}}=0.293$. It is determined by ensuring that the cavitation number in the model test at $0.8 R$ (propeller radius) at 12 o'clock is equal to that in full scale.

At the propeller design phase, the target rotation speed of a propeller is traditionally fixed because the main engine has been given and thus all the designed propellers should have close thrust at the target rotation speed. For this, the camber of the Propeller B blade is reduced to ensure that it generates equal thrust when operating at the same rotation speed as Propeller A. Fig. 3 displays the open water characteristics obtained by open water model test. The operating point in the cavitation test approximately corresponds to the advance ratio of $J=0.63$. It can be seen that the two propellers have approximately the same thrust around the operation point. Therefore, in the cavitation test, the two propellers are considered to be operating at the same cavitation number.

Tab. 1. Principle dimensions of the propellers

\begin{tabular}{|l|c|c|c|}
\hline \multicolumn{1}{|c|}{ Item } & Unit & Propeller A & Propeller B \\
\hline Diameter & $\mathrm{m}$ & 6.64 & 6.64 \\
Expanded blade area ratio & - & 0.57 & 0.57 \\
Pitch ratio at 0.7R & - & 0.850 & 0.880 \\
Chord length at 0.7R & $\mathrm{m}$ & 1.668 & 1.668 \\
Blade number & - & 5 & 5 \\
\hline
\end{tabular}
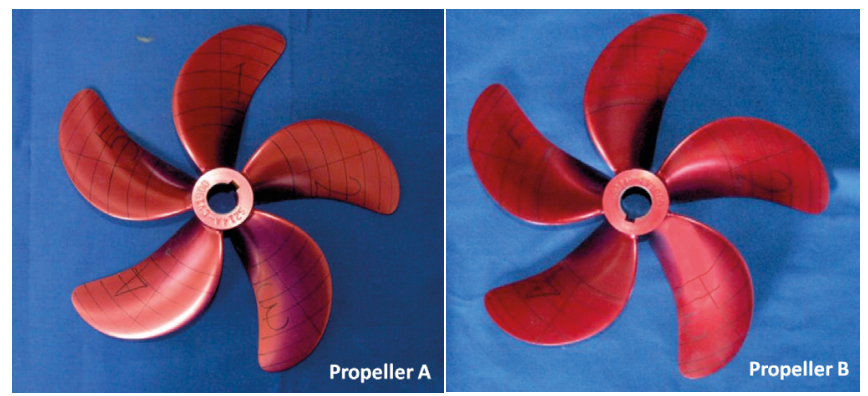

Fig. 1. Photos of the propeller models used in the experiment

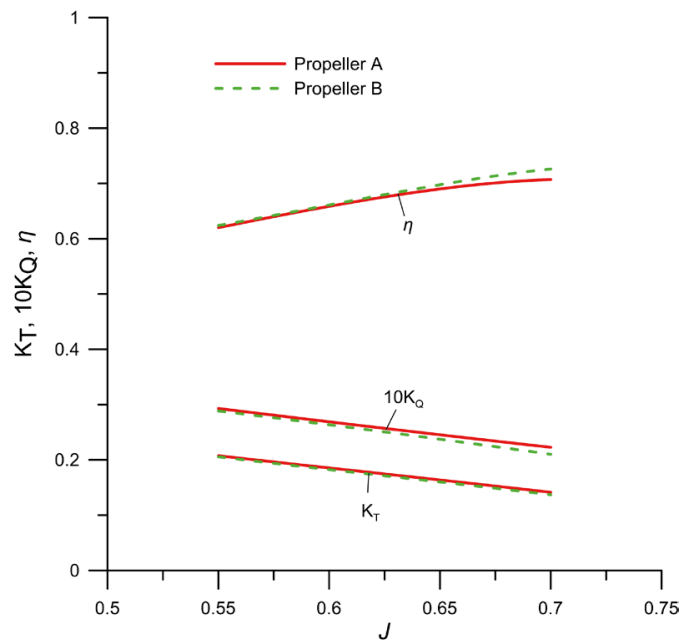

Fig. 3. Open water characteristics of the two propellers

\section{COMPUTATIONAL DETAILS}

\section{NUMERICAL MODELS}

The Schnerr-Sauer cavitation model is used here to deal with the cavitation evolution, based on a reduced RayleighPlesset (RP) equation and neglecting the influence of bubble growth acceleration, viscous effects and surface tension effects. The Schnerr-Sauer cavitation model allows the bubble growth rate and collapse rate for both single-component materials and multi-component materials to be scaled. In the Schnerr-Sauer cavitation model, the single-component cavitation bubble growth rate is estimated using the inertiacontrolled growth model:

$$
\left(\frac{d R}{d t}\right)^{2}=\frac{2}{3}\left(\frac{P_{s a t}-P_{\infty}}{\rho}\right)
$$

where $P_{\text {sat }}$ is the saturation pressure corresponding to the temperature at the bubble surface, $P_{\infty}$ is the pressure of the surrounding liquid and $\rho$ is the liquid density.

RANS method is used and the governing equations consists of continuity equation and momentum conservation equations, which can be written as:
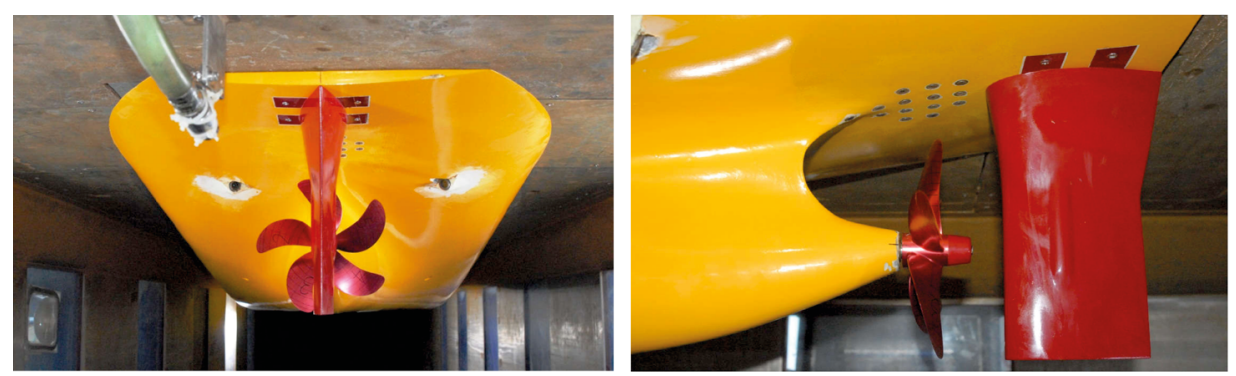

$$
\frac{\partial \bar{u}_{i}}{\partial x_{i}}=0
$$

Fig. 2. Set-up of the cavitation model test 
$\frac{\partial}{\partial t}\left(\rho \bar{u}_{i}\right)+\frac{\partial}{\partial x_{j}}\left(\rho \bar{u}_{i} \bar{u}_{j}\right)=-\frac{\partial \bar{P}}{\partial x_{i}}+\frac{\partial}{\partial x_{j}}\left[\mu\left(\frac{\partial \bar{u}_{i}}{\partial x_{j}}+\frac{\partial \bar{u}_{j}}{\partial x_{i}}\right)\right]+\frac{\partial}{\partial x_{j}}\left(-\rho \overline{\bar{u}_{i} \bar{u}_{j}^{\prime}}\right)+\bar{f}_{i}$

where: $i$ and $j$ are the coordinate components, $u$ denotes the velocity, $\rho$ is the fluid density, $\mu$ is the viscosity of the fluid, $P$ is the pressure, $f$ is the body force and $\rho \overline{u_{i}^{\prime} u_{j}^{\prime}}$ is the Reynolds stress term, which can be closed based on turbulence models. The solution of the flows in this work is based on a commercial CFD code, STAR-CCM+, which is widely used in the marine industry ${ }^{[5,13,15,16]}$ and has proved to be a reliable numerical tool for the analysis of marine hydrodynamics. Finite volume method (FVM) is adopted in STAR-CCM+ to discretize the governing equations. A second-order upwind scheme is used for the convective term. The cavitation interface is captured based on the volume of fluid (VOF) method.

\section{COMPUTATIONAL DOMAIN AND GRID SYSTEM}

The computational domain is a cube, in which a cylinder subdomain is generated to handle the propeller rotation using a sliding mesh. The dimensions of the computational domains are depicted in Fig. 4. The mesh is generated using a STAR-CCM+ automated mesh facility, which can generate unstructured hexahedral cells based on the Cartesian cutcell method. Prism layers are also generated on the ship and propeller surfaces to compute the boundary layer flow moreaccurately. The target wall normal distance of the prismatic first layer cell is $y+=2$ on blades and $y+=40$ on the hull. Fig. 5 displays the grid system for the simulations. Multiple refinement levels are used near the hull and blades to achieve a reasonable mesh distribution. The finest meshes are distributed along with the leading and trailing edges of the blades. The grid in the regions around the blade tips is also refined to capture the cavitation. The total cell number generated is 8.94 million. The time step is set to, corresponding to the blade rotating angle of 0.25 degrees in a time step.

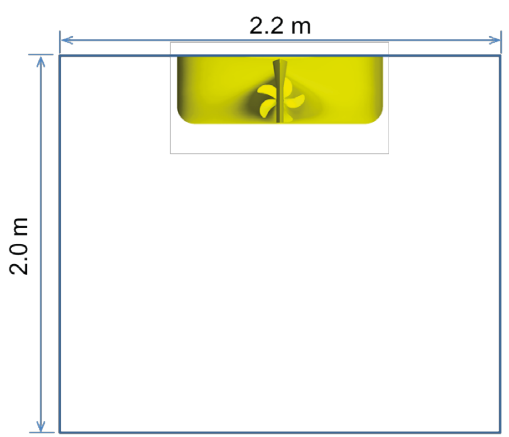

Fig. 4. Sketch of the computational domain

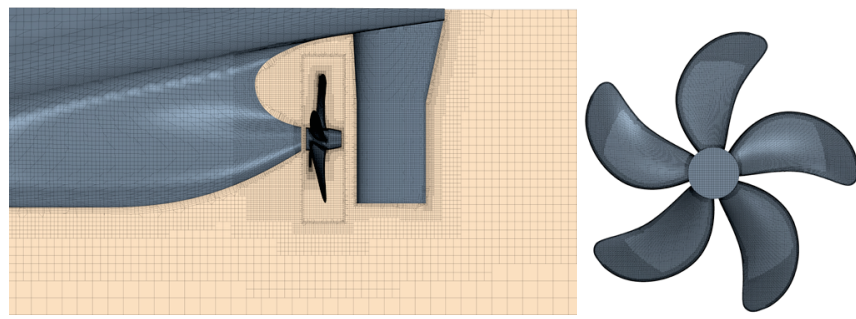

Fig. 5. Grid system for the simulations

\section{RESULTS AND DISCUSSION}

\section{GRID REFINEMENT}

Grid refinement is crucial for capturing flow details in CFD simulations. Shin et al. (2018) [17] studied an adaptive grid refinement based on Q-criterion and showed a remarkable improvement in the capturing of tip vortex cavitation. Viitanen et al. (2020) [18] also exhibited the significant grid resolution dependency of tip vortex cavitation for an open water propeller. This section discusses the grid sensitivity of the present numerical approach. Three set of grids are used here: coarse, medium and fine. The refining zones are the region around the blade tips (see Fig. 6). The cell numbers of the three grids are 8.94 million, 13.1 million and 21.1 million, respectively. The refinement ratio is 2 . Thus, the grid cell number increases considerably as the spatial resolution increases. Therefore, only one blade tip is refined in the fine grid with respect to the medium mesh to control the total grid number at an acceptable level. A similar refinement strategy was applied by Asnaghi et al. (2018) [19]. The spatial resolutions for the three grids are $0.24 \mathrm{~mm}, 0.12 \mathrm{~mm}$ and $0.06 \mathrm{~mm}$, respectively. A detailed comparison of the grid density between the refined mesh and the original mesh is shown in Fig. 7.

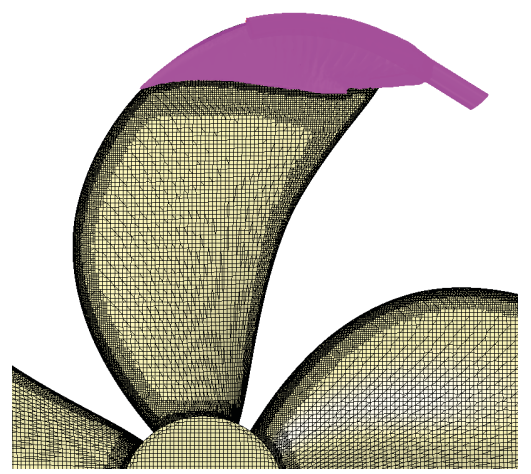

Fig. 6. Refining zones around the blade tips

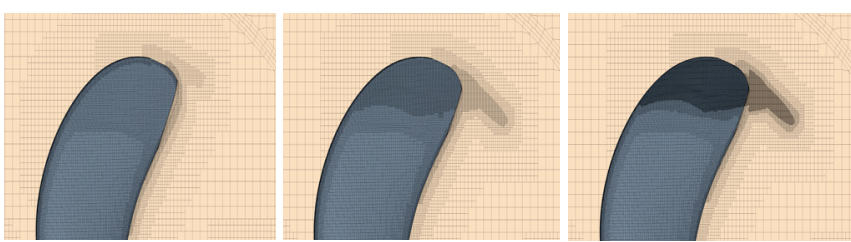

Fig. 7. Mesh distributions near the blade tips. Left: coarse grid; middle: medium grid; right: fine grid

Fig. 8 presents the cavitation patterns of a single blade of Propeller A based on different grids. Sheet cavitation is observed and gradually merges with the tip vortex cavitation. The sheet cavitation results are not sensitive to the spatial resolution. However, the simulation of the tip vortex cavitation is remarkably improved with the medium and fine grids. The coarse grid considerably underestimates the detachment of the tip vortex cavitation and gives a smaller 
extent of the tip vortex cavitation. The survival distance of the tip vortex cavitation in the refined grid is markedly longer. This is mainly because the tip vortex is quite small and more sensitive to the local low pressure in its core. Its inception is complicated and is closely related to the local flow features as well as the size of the air nuclei in the inflow. The tip vortex cavitation is unstable and dynamic as it deforms while propagating downstream under the influence of the evolution of the tip vortex. Fig. 9 shows the deformation of the tip vortex cavitation based on the medium grid, where the roll-up behaviour caused by the velocity of the tip vortex is clearly seen. The strength of the tip vortex is strongly dependent on the load distribution along the radial direction of the blade. The computed propeller has a light load near the tip, producing weak tip vortices.

Fig. 10 provides a quantitative comparison between the three grids based on vapour volume. It can be seen that the coarse grid yields a smaller vapour volume and the results of the medium and fine grids are close. As there is no major difference between the medium and fine grids in terms of cavitation shape and extent, the medium grid is adopted in the following discussion.
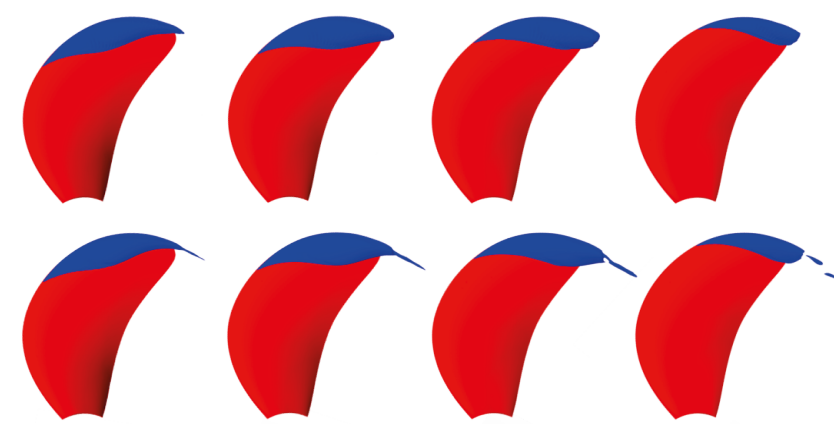

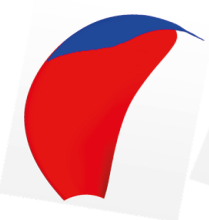

$10^{\circ}$

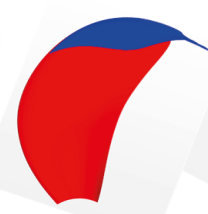

$20^{\circ}$

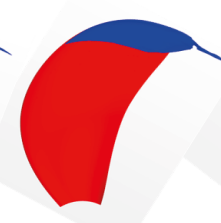

30

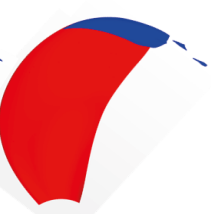

$40^{\circ}$

Fig. 8. Cavitation patterns for different grids. Top: coarse grid; middle: medium grid; bottom: fine grid.

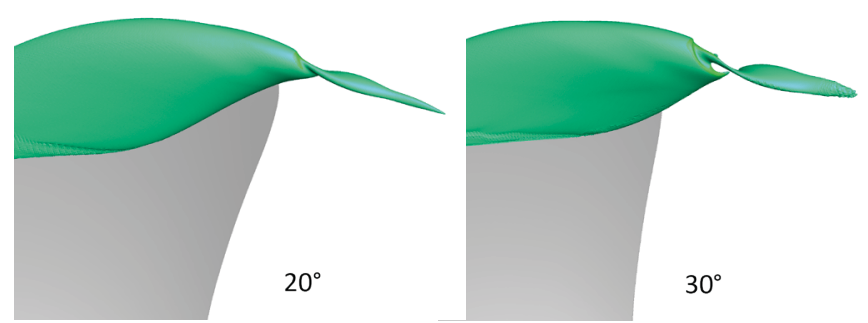

Fig. 9. Tip cavitation evolution

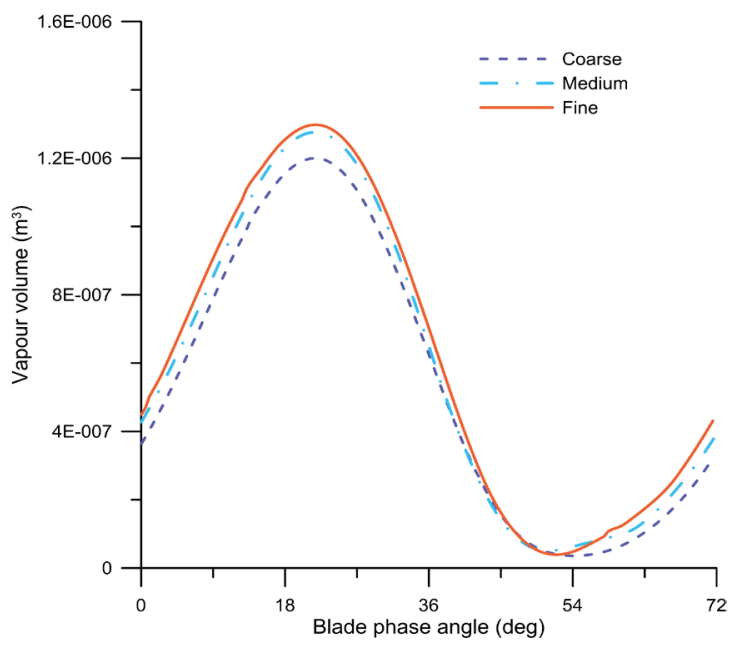

Fig. 10. Variations in vapour volume versus blade phase angle using different grids

\section{TIME STEP SENSITIVITY}

For unsteady flow, time step is an important parameter because it represents the temporal resolution. Perali et al. (2016) [20] have shown that cavity volume and pressure fluctuation are sensitive to time steps. A small time step is better able to capture the cavitation dynamics, but it increases the computational time as more time steps are needed. From an engineering designer's point of view, the time step size used should be as large as possible while sufficiently small to capture the concerned flow feature. Therefore, it is worth analyzing the effect of time step on different cavitation phenomena so that designers can choose the appropriate time step size for specific cavitation flows.

According to the CFD application guidelines of the 26th International Towing Tank Conference [21], the time step size for a rotating propeller should ensure at least 200 time steps per revolution. However, a simulation of cavitating flow usually requires a much smaller time step to capture accurately the flow detail. Local velocities and pressure are able to affect the results considerably. Therefore, here we consider three times steps $-\Delta \mathrm{t}=1 /(720 n), \Delta \mathrm{t}=1 /(1080 n)$ and $\Delta \mathrm{t}=1 /(1440 n)-$ corresponding to blade rotation angles of $1 / 2^{\circ}, 1 / 3^{\circ}$ and $1 / 4^{\circ}$ per time step, respectively. Time steps larger than $\Delta \mathrm{t}=1 /(720 n)$ are not sufficient to capture the cavitating flow, while time steps smaller than $\Delta t=1 /(1440 n)$ require too much computational time. $\mathrm{T}$

he simulation results based on the three time step sizes are shown in Fig. 11. It can be seen that the extents and patterns of sheet cavitation using different time steps are similar, whereas the survival distance of the tip vortex cavitation increases markedly as the time step size decreases. As Propeller A has a relatively smaller pitch ratio, face cavitation phenomena are observed. Fig. 12 compares the face cavitation computed using different time step sizes at the phase angle of maximum face cavitation. Again, smaller time steps yield better results. 
The results in Figs. 11 and 12 reveal that the sensitivity of cavitation calculation to time step depends on the type of cavitation being considered. Therefore, in the practical design phase, it is appropriate to use a larger time step to minimize the computational cost, when sheet cavitation is the main concern, while a small time step may be necessary to accurately predict the tip vortex cavitation and possible face cavitation.
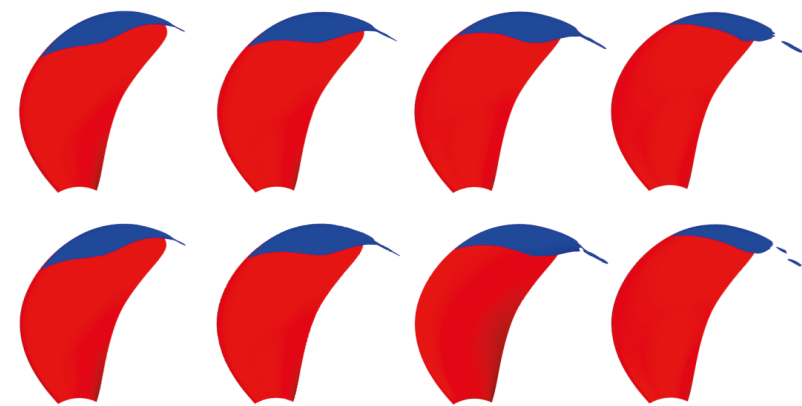

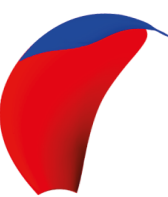

$10^{\circ}$

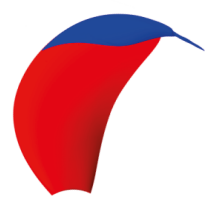

$20^{\circ}$

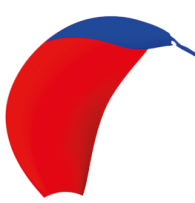

$30^{\circ}$

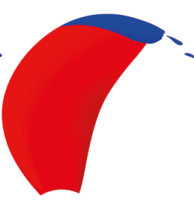

$40^{\circ}$
Fig. 11. Cavitation patterns for different time step sizes. Top: $\Delta t=1 /(720 n)$ "; medium: $\Delta t=1 /(1080 n)$; bottom: $\Delta t=1 /(1440 n) "$

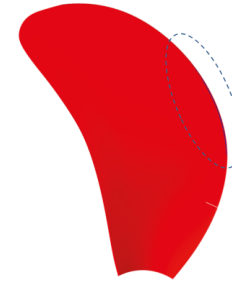

$\Delta t=1 /(720 n)$

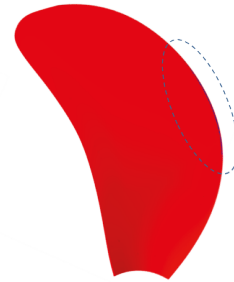

$\Delta t=1 /(1080 n)$

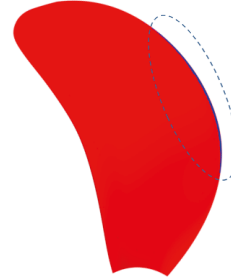

$\Delta t=1 /(1440 n)$
Fig. 12. Face cavitation at a phase angle of $246^{\circ}$ for different time step sizes

\section{EFFECTS OF TURBULENCE MODEL}

Turbulence models are commonly used to model turbulent flows in CFD simulations and the choice of turbulence model may affect the results. Various turbulence models have been proposed by researchers, the most commonly used being twoequation models such as variants of $k-\varepsilon$ and $k-\omega$. Compared with two-equation models, advanced turbulence models such as DES and LES can offer higher fidelity for flow details but need more computational time. Two-equation models provide a good compromise between computational time and accuracy. In this section, realizable $k-\varepsilon$ and SST $k-\omega$ are considered as they are the most commonly used models. The refined grid is used combined with a time step of in order to eliminate the effects of grid and time step as far as possible.
Fig. 13 provides a comparison of the cavitation patterns obtained by the two turbulence models. Overall, the cavitation extent and its variation with phase angle obtained based on the realizable $k-\varepsilon$ and SST $k$ - $\omega$ are quite similar. In detail, the realizable $k-\varepsilon$ slightly underestimates the area of the sheet cavitation compared with SST $k$ - $\omega$ and shows a slight difference in terms of the tip vortex cavitation. Fig. 14 shows the face cavitation of the two turbulence models; no evident differences between them can be observed. The results shown in Figs. 15 and 16 imply that the turbulence models have slight effects on cavitation prediction.

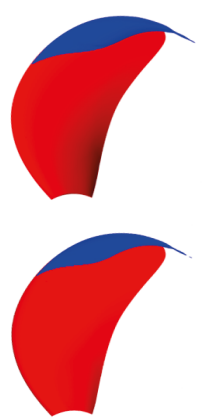

$10^{\circ}$
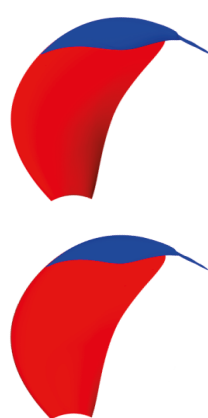

$20^{\circ}$
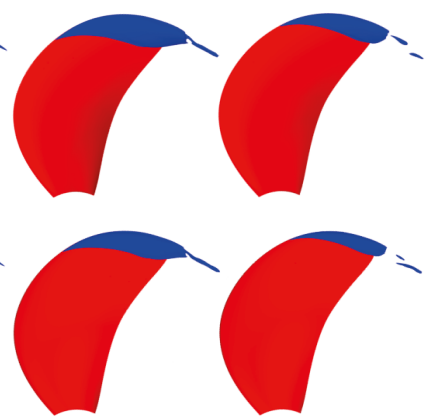

$30^{\circ}$ $40^{\circ}$
Fig. 13. Comparison of cavitation patterns between SST $k-\omega$ (top) and realizable $k-\varepsilon$ (bottom)
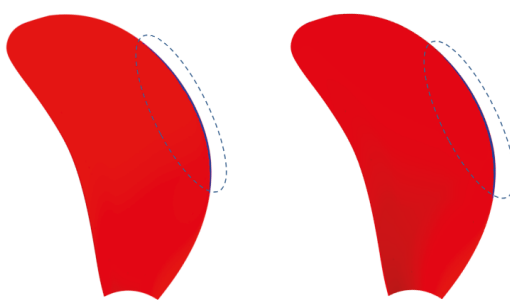

Fig. 14. Comparison of face cavitation between SST $k$ - $\omega$ (left) and realizable $k-\varepsilon$ (right)

\section{RANKING OF PROPELLERS}

The ability of RANS to rank the two propellers is analyzed in this section. Based on the above analysis, a medium grid with a time step of is adopted here. Figs. 15 and 16 show the experimental and numerical cavitation patterns at different phase angles of Propeller A and Propeller B, respectively. The level of agreement between the experimental and CFD patterns are quite good in terms of cavitation extent and location, especially for larger phase angles. Nevertheless, the tip vortex cavitation is underestimated in CFD. This may be due to the underestimation of the tip vortex's strength. Advanced turbulence models, such as DES or LES, may be able to yield better results, but this is beyond the scope of this work. As Propeller B uses a larger pitch ratio, it shows a larger sheet cavitation extent than Propeller A, as correctly differentiated by the present CFD simulation. 
Another noteworthy difference between Propeller A and Propeller B is that face cavitation is observed on the blade of the former at a phase angle of around $246^{\circ}$. This phenomenon is also well predicted by the CFD simulation (see Fig. 17). The occurrence of face cavitation in Propeller A mainly owes to the light load of the blade at specific phase angles. Fig. 18 presents the nominal wake of the hull at the propeller plane, while Fig. 19 displays the time history of a single blade thrust coefficient. It can be seen around the phase angle of $246^{\circ}$ that the wake fraction coefficient is small and the circular velocity of the hull wake is in the same direction as the blade rotation, resulting in a trough on the blade loads. Such a mechanism was also identified by Zhang et al. (2020) [22], who discussed the effect of hull wake velocity on blade load. Face cavitation should be avoided as it may cause erosion on the blade. These results imply that RANS may be a reliable tool for predicting face cavitation.
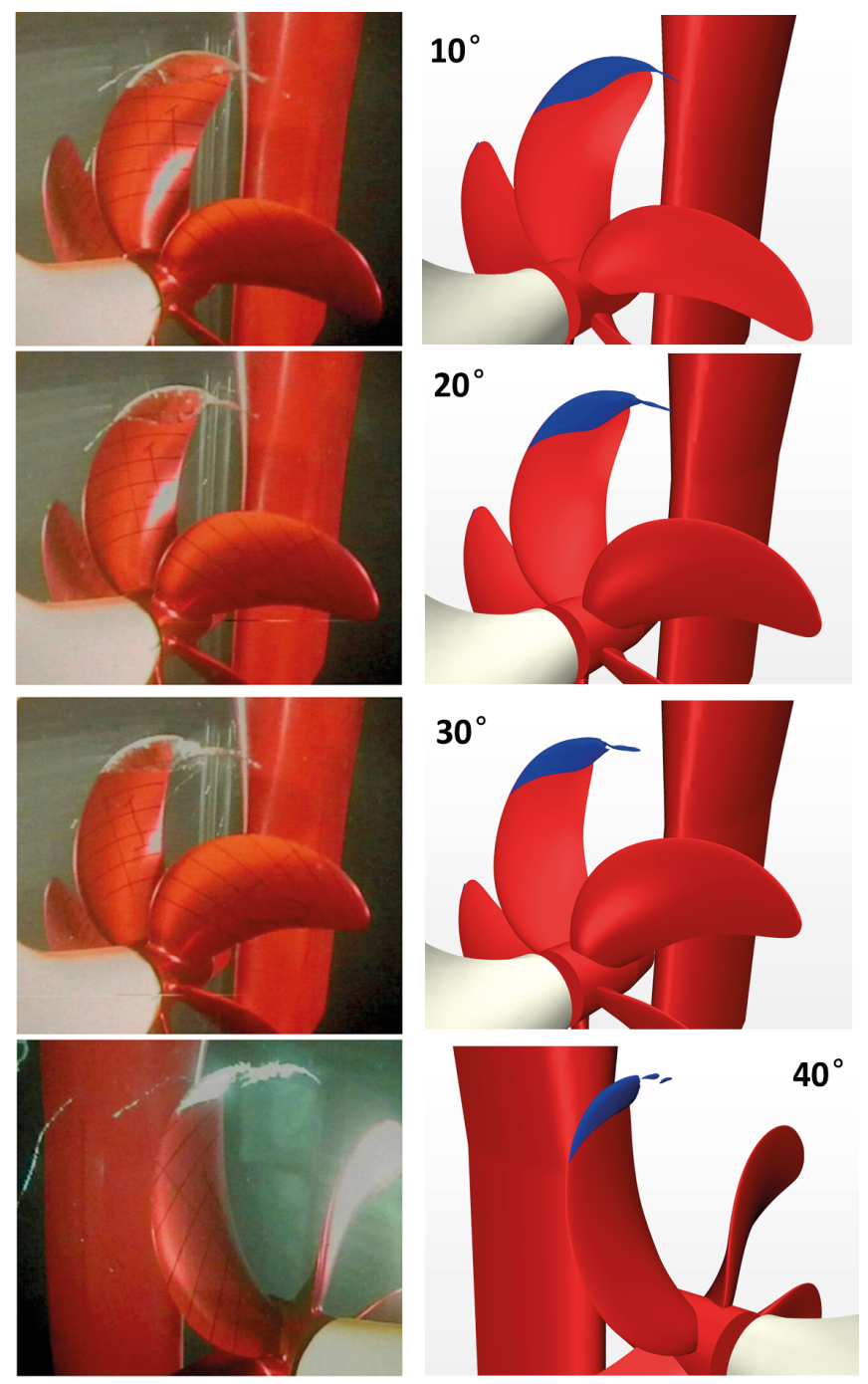

Fig. 15. Comparison of cavitation patterns between the model test and CFD for Propeller A
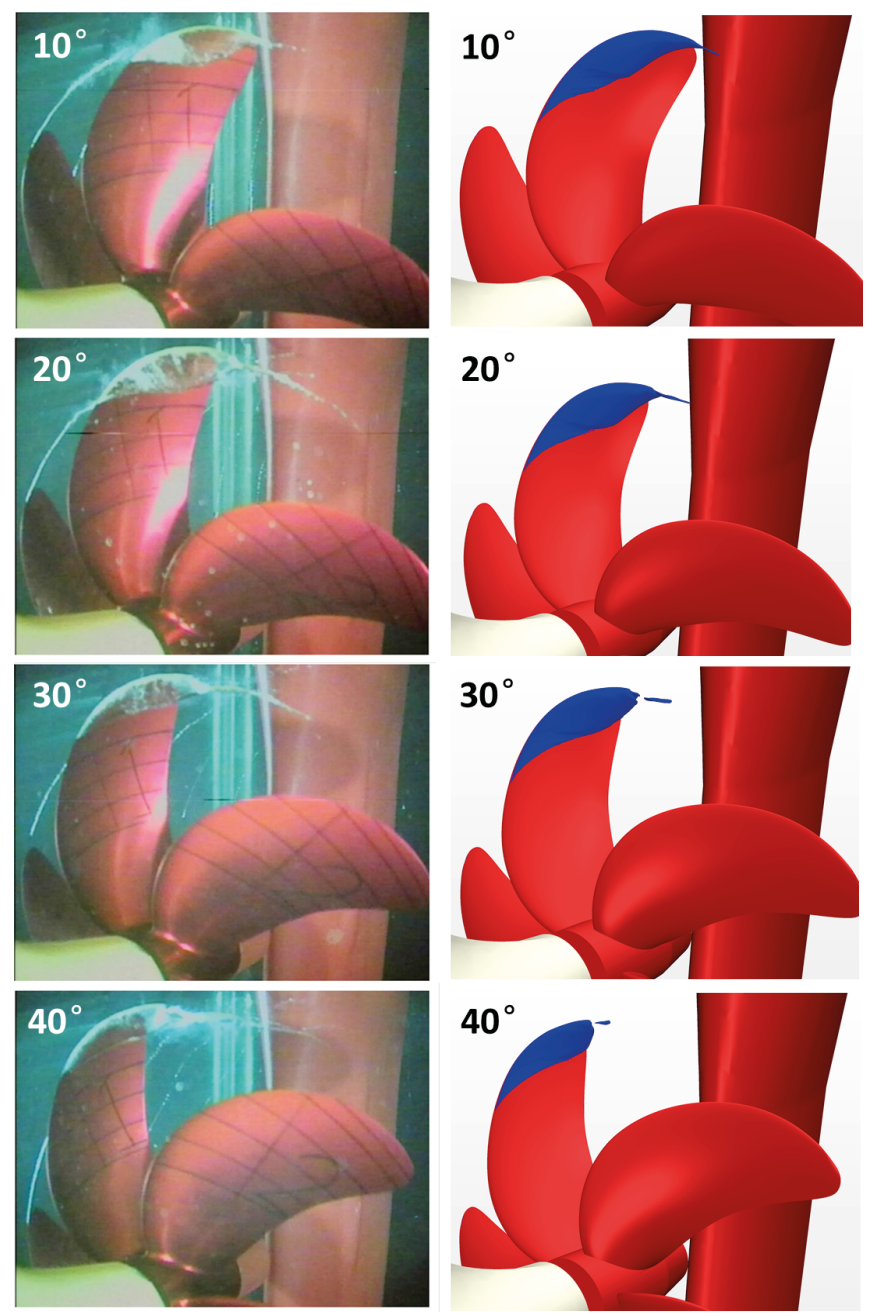

Fig. 16. Comparison of cavitation patterns between the model test and CFD for Propeller $B$
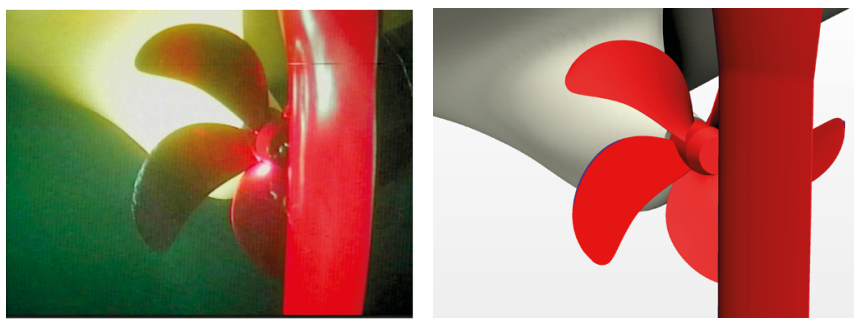

Fig. 17. Face cavitation of Propeller A obtained by the model test (left) and CFD (right) 


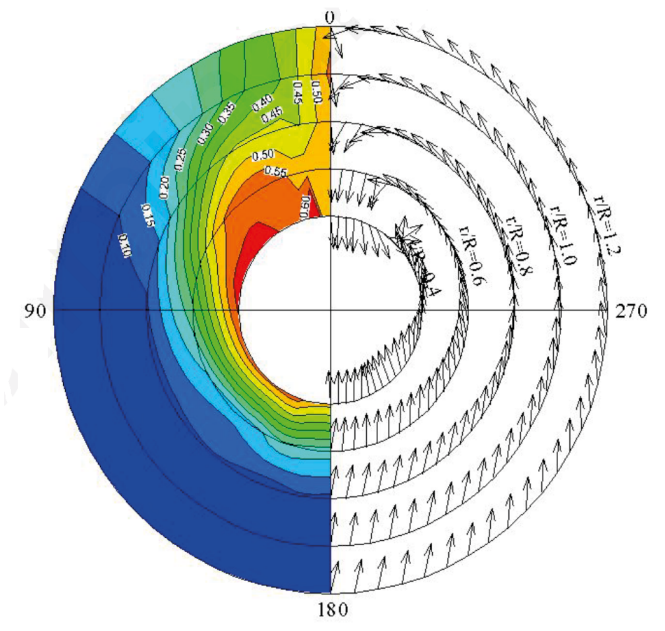

Fig. 18. Nominal wake fraction coefficient at the propeller plane

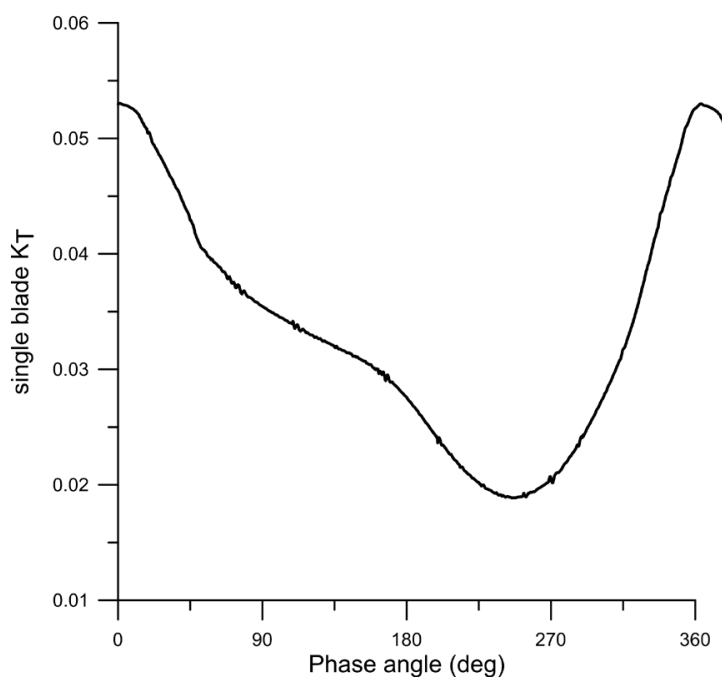

Fig. 19. Time history of a single blade thrust coefficient $\left(0^{\circ}\right.$ corresponding to 12 o'clock)

\section{CONCLUSIONS}

The aim of this study was to analyze the feasibility of the RANS method in predicting propeller cavitation. For this purpose, two propellers designed for a container vessel were investigated, differing slightly in geometry but with remarkably contrasting cavitation patterns. The analysis focused on four aspects: (i) grid sensitivity; (ii) the time step effect; (iii) the turbulence model effect; and (iv) ability to rank the two slightly different propellers. Unsteady RANS method coupled with the Schnerr-Sauer model was adopted and a model test was carried out to validate the numerical results.

Refining the grid in the cavitating region was found to yield a better prediction of the tip vortex cavitation but did not markedly affect the sheet cavitation results. Varying time steps were used to show the effect of time step size. Time steps were found to have a slight effect on the sheet cavitation pattern and a remarkable effect on both the survival of the tip vortex cavitation and the prediction of face cavitation. Moreover, two turbulence models - realizable $k-\varepsilon$ and sst $k-\omega$ - were compared, yielding similar cavitation results, implying that the cavitation prediction based on RANS was not sensitive to the turbulence models.

CFD has been found to provide good predictions of the cavitation patterns of the two propellers compared with the experimental observations in terms of cavitation extent and variation by phase angle. The differences yielded by the experimental observations in terms of cavitation extent between the two propellers were well captured by the CFD simulation. A face cavitation that was experimentally observed on Propeller A but not on Propeller B was also effectively simulated by CFD.

Author Contributions: conceptualization: Y.X.Z.; methodology: Y.X.Z.; software: Y.X.Z.; validation: Y.X.Z., X.P.W. and M.Y.L.; formal analysis: Y.X.Z., G.P.Z. and J.Z.; investigation: Y.X.Z. and J.Z.; resources: Y.X.Z., X.P.W. and J.Z.; data curation: M.Y.L.; writing-original draft preparation: Y.X.Z.; writing-review and editing: Y.X.Z., G.P.Z. and J.Z.; visualization: M.Y.L. All authors have read and agreed to the published version of the manuscript.

Conflicts of Interest: The authors declare no conflict of interest.

\section{REFERENCES}

1. F. A. Pereira, F. S. Felice, and F. Salvatore, "Propeller cavitation in non-uniform flow and correlation with the near pressure field," Journal of Marine Science and Engineering, vol. 4, pp. 1-21, 2016.

2. K., Shiraishi, Y. Sawada, D. Arakawa, and K. Hoshino, "Experimental estimation for pressure fluctuation on ship stern induced by cavitating propeller using cavity shape measurements," in Proceedings of the 10th International Symposium on Cavitation - CAV2018, Baltimore, USA, 2018.

3. A. Asnaghi, U. Svennberg, and R. E. Bensow, "Numerical and experimental analysis of cavitation inception behaviour for high-skewed low-noise propellers," Applied Ocean Research, vol. 79, pp. 197-214, 2018.

4. F. Salvatore, H. Streckwall, and T. V. Terwisga, "Propeller cavitation modelling by CFD - results from the VIRTUE 2008 Rome Workshop," in Proceedings of the First International Symposium on Marine Propulsors Smp'09, Trondheim, Norway, 2009.

5. G. Vaz, D. Hally, T. Huuva, N. Bulten, et al., "Cavitating flow calculations for the E779A propeller in open water and behind conditions: code comparison and solution validation," in Proceedings of the 4th International 
Symposium on Marine Propulsors Smp'15, Austin, Texas, USA, 2015.

6. N. Yilmaz, M. Atlar, P. A. Fitzsimmons, and N. Sasaki, "Computational fluid dynamic investigations of propeller cavitation in the presence of a rudder," in Proceedings of the 3rd International Symposium on Naval Architecture and Maritime, Istanbul, Turkey, 2018.

7. L. Wang, C. Guo, P. Xu, and Y. Su, "Analysis of the performance of an oscillating propeller in cavitating flow," Ocean Engineering, vol. 164, pp. 23-39, 2018.

8. N. Sakamoto, and H. Kamiirisa, H, "Prediction of near field propeller cavitation noise by viscous CFD with semiempirical approach and its validation in model and full scale," Ocean Engineering, vol. 168, pp. 41-59, 2018.

9. S. Gaggero, G. Tanil, D. Villa, M. Viviani, F. Miglianti, P. Ausonio, P. Travi, G. Bizzarri, and F. Serra, "Propeller geometry optimization for pressure pulses reduction: an analysis of the influence of the rake distribution," in Proceedings of the Fifth International Symposium on Marine Propulsors smp'17, Espoo, Finland, 2017.

10. J. Hur, H. Kim, and H. Lee, "Numerical study on the effect of turbulence and cavitation model for propeller induced hull pressure fluctuation," in Proceedings of the 10th International Symposium on Cavitation - CAV2018 Baltimore, Maryland, USA, 2018, pp. 834-837.

11. S. Ando, K. Kimura, K. Segawa, and K. Yamamoto, "Study on the hybrid method of CFD and bubble dynamics for marine propeller cavitation noise prediction," Proceedings of the 10th International Symposium on Cavitation - CAV2018 Baltimore, Maryland, USA, 2018, pp. 958-963.

12. C. Zheng, D. Liu, and H. Huang, "The numerical prediction and analysis of propeller cavitation benchmark tests of YUPENG ship model," Journal of Marine Science and Engineering, vol. 7, p. 387, 2019.

13. O. Usta, and E. Korkut, "A study for cavitating flow analysis using DES model," Ocean Engineering, vol. 160, pp. 397$411,2018$.

14. H. Y. Cheng, X. R. Bai, X. P. Long, B. Ji, X. X. Peng, and M. Farhat, "Large eddy simulation of the tip-leakage cavitating flow with an insight on how cavitation influences vorticity and turbulence," Applied Mathematical Modeling, vol. 77, pp. 788-809, 2020.

15. S. Gaggero, G. Tani, M. Viviani, and F. Conti, "A study on the numerical prediction of propellers cavitating tip vortex," Ocean Engineering, vol. 92, pp. 137-161, 2014.
16. Y. X. Zhang, X. P. Wu, Z. Y. Zhou, X. K. Cheng, and Y. L. $\mathrm{Li}$, "A numerical study on the interaction between forward and aft propellers of hybrid CRP pod propulsion systems," Ocean Engineering, vol. 186, p. 106084, 2019.

17. K. W. Shin, and P. Andersen, "CFD analysis of propeller tip vortex cavitation in ship wake fields," in Proceedings of the 10th International Symposium on Cavitation - CAV2018, Baltimore, USA, 2018.

18. V. Viitanen, T. Siikomen, and A. Sanchez-Caja, "Cavitation on model- and full-scale marine propellers: steady and transient viscous flow simulations at different Reynolds numbers," Journal of Marine Science and Engineering, vol. 8, p. 141, 2020.

19. A. Asnaghi, U. Svennberg, and R. E. Bensow, "Numerical and experimental analysis of cavitation inception behaviour for high-skewed low-noise propellers," Applied Ocean Research, vol. 79, pp. 197-214, 2018.

20. P. Perali, T. Lloyd, and G. Vaz, "Comparison of uRANS and BEM-BEM for propeller pressure pulse prediction: E779A propeller in a cavitation tunnel," in Proceedings of the 19th Numerical Towing Tank Symposium, Nantes, France, 2016.

21. International Towing Tank Conference (ITTC), “The specialist committee on computational fluid dynamicsfinal report and recommendations to the 26th ITTC," in 26th ITTC, vol. 2, pp. 337-377, 2011.

22. Y. X. Zhang, K. Chen, and D. P. Jiang, "CFD analysis of the lateral loads of a propeller in oblique flow," Ocean Engineering, vol. 202, p. 107153, 2020. 


\section{CONTACT WITH THE AUTHORS}

\section{Yuxin Zhang}

e-mail:yxinzhang@foxmail.com

Shanghai Merchant Ship Design and Research Institute,

2633 Zu Chong Zhi Road, 201203 Shanghai,

\section{China}

\section{Xiao-ping Wu}

e-mail: otwq@163.com

Shanghai Merchant Ship Design and Research Institute, 2633 Zu Chong Zhi Road, 201203 Shanghai,

\section{China}

\section{Ming-yan Lai}

e-mail: laimingyan2008@163.com

Shanghai Merchant Ship Design and Research Institute, 2633 Zu Chong Zhi Road, 201203 Shanghai,

\section{China}

\section{Guo-ping Zhou}

e-mail: zgp716@163.com

Shanghai Merchant Ship Design and Research Institute, 2633 Zu Chong Zhi Road, 201203 Shanghai,

\section{CHINA}

\section{Jie Zhang}

e-mail:zhangjie@shmtu.edu.cn

Shanghai Maritime University,

1550 Haigang Ave, 201306 Shanghai,

\section{China}

